\title{
Clinical Relevance of Gastroesophageal Cancer Associated SNPs for Oncologic Outcome After Curative Surgery
}

\author{
Jin-On Jung, $\mathrm{MD}^{1,4}$ (ㅁ), Naita Maren Wirsik, $\mathrm{MD}^{1,4}$, Henrik Nienhüser, MD ${ }^{1}$, Leila Peters, $\mathrm{MD}^{1}$, \\ Beat Peter Müller-Stich, MD ${ }^{1}$, Timo Hess, MSc $^{2,3}$, Vitalia Schüller, MSc ${ }^{2}$, Johannes Schumacher, MD ${ }^{2,3}$, and \\ Thomas Schmidt, MD, $\mathbf{P h D}^{1,4}$ (i)
}

${ }^{1}$ Department of General, Visceral and Transplantation Surgery, University of Heidelberg, Heidelberg, Germany; ${ }^{2}$ Institute of Human Genetics, University of Bonn, Bonn, Germany; ${ }^{3}$ Institute of Human Genetics, University of Marburg, Marburg, Germany; ${ }^{4}$ Present address: Department of General, Visceral and Transplantation Surgery, University Clinic Cologne, Cologne, Germany

\begin{abstract}
Background. Gastric and esophageal cancers are malignant diseases with rising importance in Western countries. To improve oncologic outcome after surgery, it is essential to understand the relevance of germline mutations. The aim of the study was to identify and distinguish clinically relevant single-nucleotide polymorphisms (SNPs).

Patients and Methods. In total, 190 patients with curative oncological resections of gastric and distal esophageal adenocarcinomas at Heidelberg University Hospital were eligible for this study. Outcome differences were determined for each SNP by analysis of clinical variables, survival, and mRNA expression levels.

Results. Significant survival differences were found on univariate analysis for usual prognostic variables (such as pTNM) and for six SNPs. On multivariate survival analysis, the SNPs rs12268840 (intron variant of $M G M T, p=$ 0.045 ) and rs 9972882 (intron variant of STARD3 and eQTL of $P G A P 3, p=0.030$ ) were independent and significant survival predictors along with $\mathrm{R}$ status and $\mathrm{pT} / \mathrm{pN}$ category. Group TT of rs12268840 had the highest rate of second primary carcinoma $(30.4 \%, p=0.0003)$, lowest expression
\end{abstract}

(C) The Author(s) 2021

First Received: 10 June 2021

Accepted: 18 August 2021;

Published Online: 16 September 2021

J.-O. Jung, MD

e-mail: jin-on.jung@uk-koeln.de

T. Schmidt, MD, PhD

e-mail: Thomas.Schmidt1@uk-koeln.de of $M G M T$ based on cis-eQTL analysis in normal gastroesophageal tissue $\left(p=1.99 \times 10^{-17}\right)$, and worst oncologic outcome. Group AA of rs9972882 had the highest rate of distant metastases pM1 $(42.9 \%, p=0.0117)$, highest expression of PGAP3 $\left(p=1.29 \times 10^{-15}\right)$, and worst oncologic outcome.

Conclusions. Two intron variant SNPs of MGMT and STARD3 were identified that were significant survival predictors and may influence tumor biology. The data indicate that DNA methylation (MGMT) and malfunction of GPI anchoring (PGAP3) are distinct mechanisms that are relevant for tumor progression and relapse.

Cancers of the stomach and esophagus are devastating diseases accounting for nearly $9 \%$ of all malignancies. ${ }^{1}$ In Western countries, more than two-thirds of gastric cancer cases are diagnosed at an advanced stage with a poor 5-year survival rate of about $25 \% .^{2}$ Moreover, the incidence of esophageal adenocarcinoma has been rapidly increasing. ${ }^{3}$ Gastroesophageal reflux disease (GERD) and Barrett's esophagus (BE) apart from obesity and tobacco smoking were identified as significant risk factors, ${ }^{4}$ especially for adenocarcinoma of the gastroesophageal junction (GEJ). Preexisting GERD leads to a 6.2-fold risk for GEJ, ${ }^{3}$ while antireflux therapies, physical activity, breastfeeding, nonsteroidal antiinflammatory drugs (NSAIDs), and HMGCoA reductase inhibitors (statins) are inversely associated risk factors. Similar risk factors could be identified for gastric cancer (GC) as GERD bears a two- to fourfold risk for GC. ${ }^{5}$ Furthermore, it is known that chronic infection with Heliobacter pylori is another risk for GC. 
Genome-wide genotyping is a frequently used technique that has been widely practiced in the past. Recently, various single-nucleotide polymorphisms (SNPs) were identified that associate with either GERD, BE, GEJ, or GC. ${ }^{6}$ Most studies utilize high case numbers and hundreds of thousands of SNPs, but do not address specific clinical parameters other than the rough differentiation between case and control groups. However, it is necessary to establish ties between genotyped data and oncological outcome by making use of systematic follow-up on a welldifferentiated cohort. While the number of allegedly relevant SNPs increases, it becomes harder to maintain an overview of those SNPs, which might also have a clinical significance and lead back to molecular pathways that are actually relevant. By correlating genotyped and imputed data with oncologic outcome parameters, we aim to identify clinically relevant loci.

\section{PATIENTS AND METHODS}

\section{Data Collection}

Eligible subjects of this study were patients with GC or GEJ who underwent oncological resection of gastric or esophageal cancer between 2008 and 2017 at Heidelberg University Hospital, Department of General Surgery. All patients provided written consent for data collection and analysis. Patients were excluded who deceased during the early postoperative phase or in-hospital as oncologic outcome was assessed. The trial protocol was approved by the ethics committee at the University of Heidelberg and was performed in accordance with the Declaration of Helsinki and Good Clinical Practices as well as local ethics and legal requirements.

\section{Genotyping}

Prior to oncological resection, genomic DNA was extracted from peripheral blood leukocytes. Genotyping was performed by collaborating partners at the Department of Human Genetics, University of Bonn, Germany. It was accomplished for all patients using Infinium OmniExpress, Infinium OmniExpressExome, and Infinium Omni2.5Exome BeadChips (by Illumina) according to the manufacturer's protocol at the University of Bonn. The overlap of the SNP content was subjected to initial quality control performed with PLINK v1.90b6.6. Samples with genotype call rate less than 97\%, discrepancies in sex, divergent ancestry from the CEU HapMap 2010 population, and related samples were excluded from further analysis.

\section{Imputation}

All unambiguous SNPs with SNP call rate $>95 \%$, minor allele frequency $(\mathrm{MAF})>1 \%$, and Hardy-Weinberg equilibrium (HWE) $>0.001$ were used for imputation. Imputation was performed with IMPUTE2 based on 1,000 Genomes Phase 3 as reference panel. As post-imputation quality control, we excluded all variants with an information score (INFO-score) $<0.8$, HWE $<0.001$, MAF $<1 \%$, and SNP missing rate $>5 \%$ for best-guessed genotypes at posterior probability greater than 0.9 from further analysis.

\section{Histopathology and Follow-Up}

In all cases, the diagnosis of gastric and esophageal adenocarcinoma was histopathologically confirmed and the resected specimens were analyzed at the Department of Pathology, University of Heidelberg. The histopathological staging was performed according to the 8th UICC edition, including TNM categories, $\mathrm{R}$ status, and grading. ${ }^{7} \mathrm{In}$ hospital mortality and postoperative complications were recorded during hospital course. To acquire long-term survival data, all patients were systematically followed up via continuous surveys. The relevant survival data for this patient collective were gathered until October 2020.

\section{Statistical Analysis}

We extracted the genotype information from the initial PLINK files by using Gtool v0.7.5. The data were collocated using Microsoft Office Excel, and statistical tests as well as plots were calculated thereafter via GraphPad Prism 9 for Mac OS X. We defined overall survival from time of first diagnosis until death or until the most recent follow-up using the Kaplan-Meier method. Differences in survival were analyzed via Mantel-Cox (logrank) comparisons on STATA/SE 15.0 for Mac. A level of significance of $\alpha=$ 0.05 was generally considered as statistically significant. For multivariate survival analysis, we established a Cox proportional hazards model with a level of significance of $\alpha$ $<0.2$. All clinical and SNP parameters from the univariate studies with a $p$-value $<0.2$ were included. The preoperatively assessed cTNM categories were excluded since a precise histopathology was available via the final pTNM histology.

\section{Literature-Based SNP Identification}

We performed a MEDLINE search via PubMed for SNPs that were reported as being responsible for or associated with BE, GERD, GEJ, or GC in previous genomewide association studies. Search items varied and included terms such as "genome wide association study," "gastric 
cancer," "esophageal cancer," "gastroesophageal reflux," "Barrett's esophagus," "SNP," and others. As of January 2021, a MeSH search with terms "genome wide association study" in combination with either "gastric neoplasm" ( $n=122,42 \%)$, "esophageal neoplasm" $(n=117,40 \%)$, "gastroesophageal reflux" $(n=13,4 \%)$, or "Barrett's esophagus" ( $n=41,14 \%)$ showed a total result of 293 original articles. All reported SNPs were searched, and those that were available in the genotyped and imputed datasets were further analyzed.

\section{cis-eQTL Analysis in Normal Tissue}

For cis expression quantitative trait loci (cis-eQTL) analyses in normal tissue, we accessed the Genotype-Tissue Expression (GTEx) database (Version 8; Release date 18 July 2019: dbGaP Accession phs000424.v8.p2; http:// www.gtexportal.org/home/). All local genes $+/-1 \mathrm{Mb}$ of the selected SNPs were extracted based on data provided by the GENCODE project. We retrieved the summary statistics of linear regression results via GTEx eQTL Calculator and generated violin plots via GTEx eQTL Dashboard with layout modifications via Adobe Illustrator CC 2018 (Version 22.0.0).

\section{RESULTS}

\section{Comparative Study-Whole Collective, GC, and GEJ}

In total, 61 out of 190 patients $(32.1 \%)$ were diagnosed with GEJ (Siewert type I-III) and 129 patients (67.9\%) had GC. Among GEJ patients, $82.0 \%$ were male, as opposed to $58.1 \%$ of GC patients $(p=0.0017)$. GEJ patients had a higher mean BMI of $26.9 \mathrm{~kg} / \mathrm{m}^{2}$ compared with GC patients (mean BMI $25.3 \mathrm{~kg} / \mathrm{m}^{2}, p=0.0196$ ). Besides neoadjuvant therapy ( $85.2 \%$ in GEJ versus $57.4 \%$ in GC, $p=0.0001)$, there were no significant differences between GEJ and GC patients regarding usual survival predictors. The results are demonstrated in Table 1 for the whole collective and separately for GEJ and GC in comparison, showing the significant variables that were also used in the latter multivariate survival analysis. It is important to note that both tumor entities GEJ and GC did not show any significant differences in overall survival $(p=0.9417)$ and disease-free survival $(p=0.3893)$. The corresponding Kaplan-Meier curves are demonstrated in Supplementary Fig. 1a, b.

\section{Overall Survival-Clinical Parameters}

Median follow-up duration was 49.08 months (73.25 months for living cases and 20.74 months for deceased cases). The preoperative variables cT ( $p=0.0045), \mathrm{cN}(p=$ $0.0079)$, and cM category $(p<0.0001)$ were significant predictors of survival. Neoadjuvant therapy $(p=0.0229)$ and type of operation $(p<0.0001)$ were also clinical variables that were shown to have statistical significance. Finally, the histopathological parameters pT $(p<0.0001)$, $\mathrm{pN}(p<0.0001)$, $\mathrm{pM}(p<0.0001)$, and $\mathrm{R}$ status $(p<$ $0.0001)$ were significant for outcome. All results are summarized in Supplementary Table 1A,B.

\section{Overall and Disease-Free Survival-SNPs}

In total, 68 SNPs, which were identified in the literature search, were analyzed by univariate survival analysis (Supplementary Table 2A,B). Six SNPs showed significant differences on univariate overall survival (OS) analysis. The SNP IDs, genetic information, and median survival time (in months) are summarized in Table 2.

For rs12268840 (located on chromosome 10, intron variant of $M G M T$ ), 101 patients were genotyped as CC with a median OS of 62.1 months, 65 patients as TC (median OS incalculable), and 23 patients as TT with a median OS of 29.7 months ( $p=0.0119$; Fig. 1a). SNP rs9972882 is located on chromosome 17 and an intron variant of the Star-related lipid transfer domain containing 3 (STARD3). For rs9972882, 14 patients were genotyped as AA with a median OS of 20.3 months, 65 patients as AC with a median OS of 85.6 months, and 111 patients as $\mathrm{CC}$ with a median OS of 57 months ( $p=0.0214$; Fig. 1b). The Kaplan-Meier plots of the remaining four SNPs with significant survival differences in univariate analysis are shown in Supplementary Fig. 2a-d.

Figure 1c, d shows the disease-free survival (DFS) plots for both SNPs rs12268840 and rs9972882. Disease-free survival for SNP rs9972882 was worst in group AA $(n=$ 14 , median DFS = 13.5 months) compared with groups AC ( $n=65$, median DFS $=106.1$ months $)$ and CC $(n=111$, median DFS $=90.1$ months), though not significant $(p=$ $0.0675)$. Likewise, there were no significant differences in disease-free survival curves of groups TT $(n=23$, median DFS $=47.1$ months $)$ TC $(n=65$, median DFS $=121.4$ months), and CC ( $n=101$, median DFS $=90.1$ months $)$ of SNP rs12268840, with none of the groups showing any outstanding tendency according to the logrank test ( $p=$ $0.5625)$.

\section{Multivariate Regression}

To further evaluate the significance of the identified SNPs, a multivariate regression analysis was performed including the clinical prognostic factors (Table 3 ). The histological parameters pT $(p=0.020)$ and $\mathrm{pN}$ category $(p=0.001)$ as well as $\mathrm{R}$ status $(p=0.006)$ were statistically 
TABLE 1 Selection of clinical survival predictors and comparison between GC and GEJ

\begin{tabular}{|c|c|c|c|c|c|}
\hline & & $\begin{array}{l}\text { Whole collective (GEJ and } \\
\text { GC) }\end{array}$ & $\begin{array}{l}\text { Gastroesophageal junction cancer } \\
\text { (GEJ) }\end{array}$ & $\begin{array}{l}\text { Gastric cancer } \\
\text { (GC) }\end{array}$ & $\begin{array}{l}p- \\
\text { value }\end{array}$ \\
\hline \multirow[t]{3}{*}{ Tumor entity } & GEJ I/II & $48(25.2 \%)$ & $n=61$ & $n=129$ & \multirow[t]{3}{*}{-} \\
\hline & GEJ III & $13(6.8 \%)$ & $(32.1 \%)$ & $(67.9 \%)$ & \\
\hline & GC & $129(67.9 \%)$ & & & \\
\hline \multirow[t]{2}{*}{ Sex** } & Male & $125(65.8 \%)$ & $50(82.0 \%)$ & $75(58.1 \%)$ & \multirow[t]{2}{*}{0.0017} \\
\hline & Female & $65(34.2 \%)$ & $11(18.0 \%)$ & $54(41.9 \%)$ & \\
\hline \multirow{2}{*}{$\begin{array}{l}\text { BMI } \\
\left(\text { in } \mathrm{kg} / \mathrm{m}^{2}\right)^{*}\end{array}$} & Mean & 25.8 & 26.9 & 25.3 & \multirow[t]{2}{*}{0.0196} \\
\hline & $95 \% \mathrm{CI}$ & $25.2-26.5$ & $25.6-28.2$ & $24.6-26.0$ & \\
\hline \multirow{2}{*}{$\begin{array}{l}\text { Neoadjuvant } \\
\text { (R)CTx*** }\end{array}$} & No & $64(33.7 \%)$ & $9(14.8 \%)$ & $55(42.6 \%)$ & \multirow[t]{2}{*}{0.0001} \\
\hline & Yes & $126(66.3 \%)$ & $52(85.2 \%)$ & $74(57.4 \%)$ & \\
\hline \multirow[t]{7}{*}{ (y)pT category } & 0 & $10(5.3 \%)$ & $5(8.2 \%)$ & $5(3.9 \%)$ & \multirow[t]{7}{*}{0.6465} \\
\hline & $1 \mathrm{a}$ & $12(6.3 \%)$ & $1(1.6 \%)$ & $11(8.5 \%)$ & \\
\hline & $1 b$ & $19(10.0 \%)$ & $5(8.2 \%)$ & $14(10.9 \%)$ & \\
\hline & 2 & $22(11.6 \%)$ & $7(11.5 \%)$ & $15(11.6 \%)$ & \\
\hline & 3 & $91(47.9 \%)$ & $36(59.0 \%)$ & $55(42.6 \%)$ & \\
\hline & $4 a$ & $22(11.6 \%)$ & $6(9.8 \%)$ & $16(12.4 \%)$ & \\
\hline & $4 b$ & $14(7.4 \%)$ & $1(1.6 \%)$ & $13(10.1 \%)$ & \\
\hline \multirow[t]{4}{*}{ (y)pN category } & 0 & $78(41.1 \%)$ & $24(39.3 \%)$ & $54(41.9 \%)$ & \multirow[t]{4}{*}{0.9096} \\
\hline & 1 & $28(14.7 \%)$ & $9(14.8 \%)$ & $19(14.7 \%)$ & \\
\hline & 2 & $37(19.5 \%)$ & $16(26.2 \%)$ & $21(16.3 \%)$ & \\
\hline & $3 a / b$ & $47(24.7 \%)$ & $12(19.7 \%)$ & $35(27.1 \%)$ & \\
\hline \multirow[t]{2}{*}{ (y)pM category } & 0 & $161(84.7 \%)$ & $52(85.2 \%)$ & $109(84.5 \%)$ & \multirow[t]{2}{*}{0.8953} \\
\hline & 1 & $29(15.3 \%)$ & $9(14.8 \%)$ & $20(15.5 \%)$ & \\
\hline \multirow[t]{2}{*}{$\mathrm{R}$ status } & 0 & $152(80.0 \%)$ & $46(75.4 \%)$ & $106(82.2 \%)$ & \multirow[t]{2}{*}{0.3318} \\
\hline & Any $X / 1 / 2$ & $38(20.0 \%)$ & $15(24.6 \%)$ & $23(17.8 \%)$ & \\
\hline \multirow[t]{2}{*}{ Overall survival $\dagger$} & Median (months) 95\% & 54.4 & 57.0 & 52.1 & \multirow[t]{2}{*}{0.9417} \\
\hline & CI & $39.9-90.2$ & 27.8-incalc. & $38.9-94.8$ & \\
\hline \multirow{2}{*}{$\begin{array}{l}\text { Disease-free } \\
\text { survival } \dagger\end{array}$} & Median (months) 95\% & 106.1 & incalc. & 106.1 & \multirow[t]{2}{*}{0.3893} \\
\hline & $\mathrm{CI}$ & 47.1-incalc. & 15.7-incalc. & 51.4-incalc. & \\
\hline
\end{tabular}

${ }^{\dagger}$ According to logrank test

${ }^{*} p<0.05$

${ }^{* * *} p<0.01$

**** $p<0.001$

significant as well as the SNPs rs12268840 $(p=0.033)$ and rs9972882 $(p=0.041)$. In a confirmative Cox regression model, the parameters pT category $(p=0.009), \mathrm{pN}$ category $(p<0.001), \mathrm{R}$ status $(p<0.001), \operatorname{rs} 12268840(p=$ $0.045)$, and rs9972882 ( $p=0.030)$ were significant survival predictors.

\section{Characterization and Clinical Differences for rs12268840 (MGMT) and rs9972882 (STARD3)}

Prognostic parameters such as the pT $(p=0.4966), \mathrm{pN}$ ( $p=0.1675)$, and $\mathrm{pM}$ category $(p=0.5984)$ or the $\mathrm{R}$ status $(p=0.6419)$ were not different between the three groups CC, TC, and TT for SNP rs12268840. However, we found that rates of second primary carcinomas (SPC) were significantly higher in the TT group (average rate $30.4 \%$ ) as compared with $9.2 \%$ in the TC group and $4.0 \%$ in the CC group ( $p=0.0003)$. Furthermore, the three groups differed significantly in preoperatively detected Laurén type ( $p=$ 0.0243).

In total, $42.9 \%$ of patients in group AA of SNP rs9972882 had distant metastases (pM1) on final histopathological workup as opposed to $12.3 \%$ in group $\mathrm{AC}$ and $13.5 \%$ in group $\mathrm{CC}(p=0.0117)$. The remaining histological parameters pT $(p=0.1029)$ and $\mathrm{pN}$ category ( $p=0.1226)$ as well as the $\mathrm{R}$ status $(p=0.6976)$ were not significantly different between the groups, either. All 


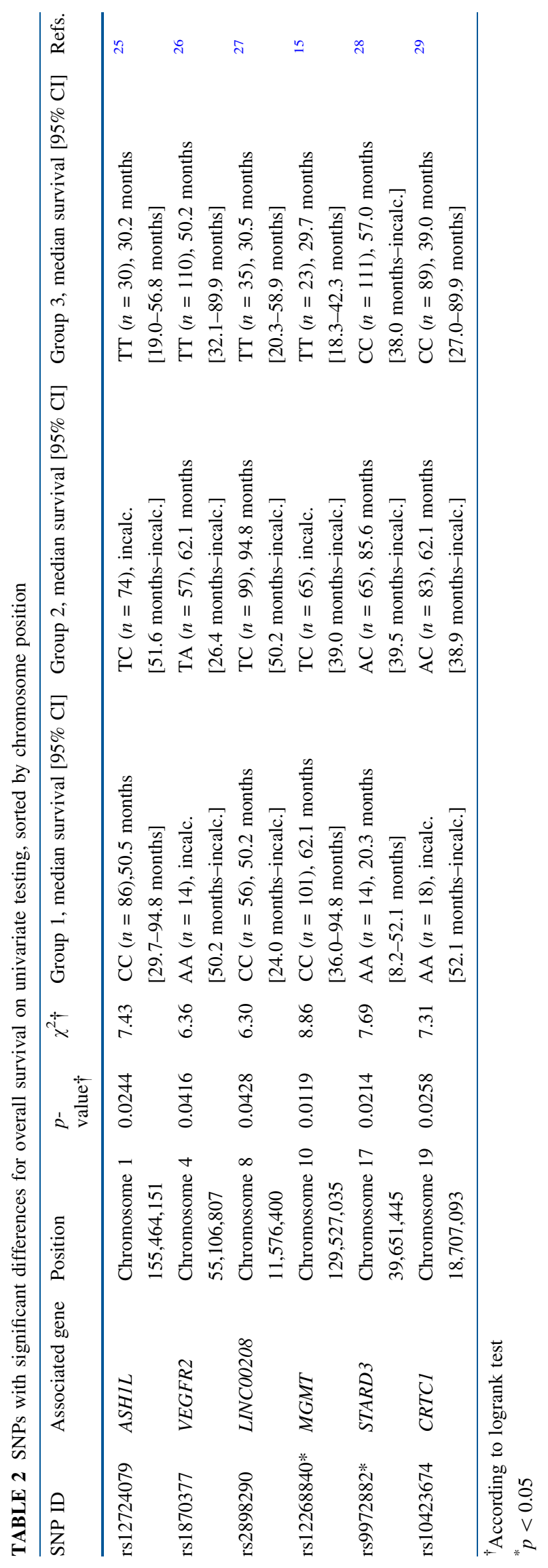


(a) Overall survival for rs12268840(MGMT)

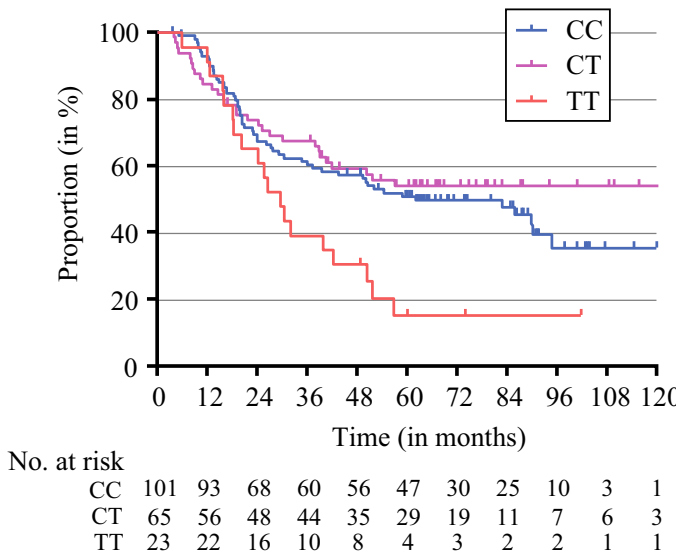

(b) Overall survival for rs9972882 (STARD3)

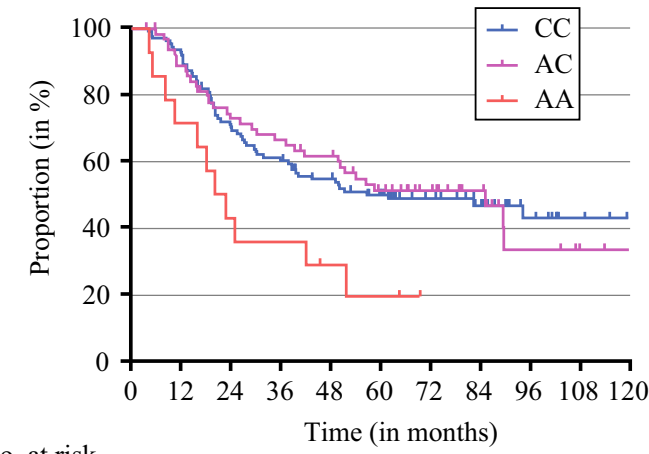

No. at risk

$\begin{array}{llllllllllll}\mathrm{CC} & 111 & 104 & 80 & 67 & 58 & 49 & 32 & 24 & 12 & 5 & 2\end{array}$

$\begin{array}{cccccccccccc}\mathrm{AC} & 65 & 57 & 46 & 42 & 38 & 29 & 20 & 13 & 6 & 4 & 2\end{array}$

FIG. 1 a, b Kaplan-Meier overall survival plots for groups CC/CT/ TT in rs12268840 (intron variant of MGMT) and for groups CC/AC/ AA in rs9972882 (intron variant of STARD3). c, d Kaplan-Meier

results for SNPs rs12268840 and rs9972882 are summarized in Supplementary Table 3A,B.

\section{Cis Expression Quantitative Trait Loci (cis-eQTL) in Normal Upper Gastrointestinal Tissue}

To assess if the above-mentioned two SNPs were also able to change the level of gene expression, we further performed cis expression quantitative trait loci (cis-eQTL) analysis for all local genes in normal gastric and esophageal tissue (mucosa, muscularis, and gastroesophageal junction). The results are presented in Supplementary Table 4A,B.

We found that rs12268840 was a highly significant ciseQTL for $M G M T$ in all upper gastrointestinal tissue types. This is supported by the normalized effect size (NES) of -0.60 in gastroesophageal junction tissue $(p=1.5 \times$ $10^{-23}$ ), meaning that the alternative allele (in this case, $\mathrm{T}$ ) (c) Disease free survival for rs12268840 (MGMT)

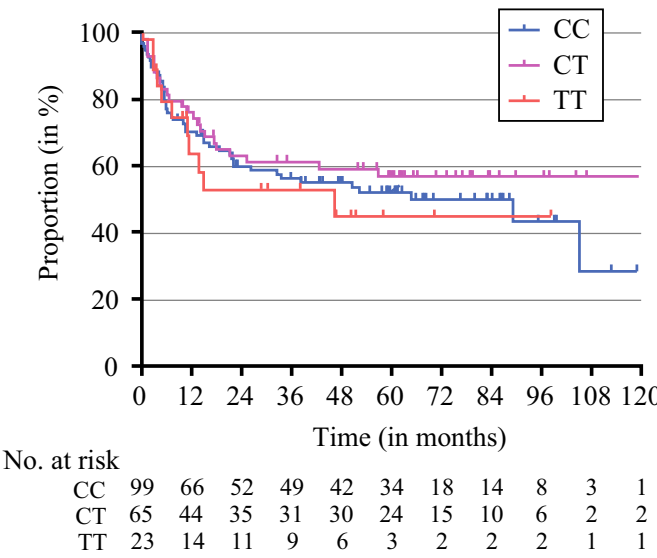

(d) Disease free survival for rs9972882 (STARD3)

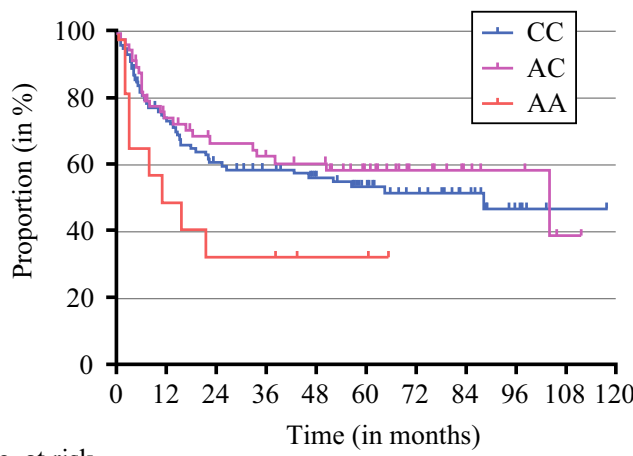

No. at risk

$\begin{array}{cccccccccccc}\text { CC } & 110 & 74 & 59 & 67 & 46 & 35 & 23 & 16 & 10 & 3 & 2 \\ \text { CT } & 64 & 43 & 35 & 42 & 30 & 24 & 12 & 9 & 5 & 2 & 1 \\ \text { TT } & 14 & 7 & 5 & 6 & 3 & 3 & 1 & 1 & 1 & 1 & 1\end{array}$

disease-free survival plots for groups CC/CT/TT in rs12268840 and for groups CC/AC/AA in rs9972882

leads to a significantly lower expression of $M G M T$ as compared with the reference allele $\mathrm{C}$. Likewise, in esophageal mucosa NES was $-0.64\left(\mathrm{p}=3.5 \times 10^{-35}\right)$, in esophageal muscularis $-0.52\left(p=3.0 \times 10^{-28}\right)$, and in gastric tissue $-0.56\left(p=2.0 \times 10^{-17}\right)$ (Fig. 2a).

rs9972882 was a significant cis-eQTL for PGAP3 in normal gastric, gastroesophageal, and esophageal muscularis tissue (but not in esophageal mucosa). NES in gastroesophageal junction tissue was $-0.15(p=3.3 \times$ $\left.10^{-6}\right)$, in esophageal muscularis $-0.17\left(p=3.4 \times 10^{-11}\right)$, and in gastric tissue $-0.23\left(p=1.3 \times 10^{-15}\right)$. In all normal upper gastroesophageal tissue types, rs9972882 did not prove to be a significant eQTL for STARD3 and ERBB2 (Her2/neu) (Fig. 2b). 
TABLE 3 Multivariate overall survival analysis, Cox regression, and successive confirmation

\begin{tabular}{|c|c|c|c|c|c|}
\hline Predictor variable & Hazard ratio & SE & Lower $95 \%$ limit & Upper $95 \%$ limit & $p$-value \\
\hline ASA score & 1.36 & 0.29 & 0.90 & 2.06 & 0.147 \\
\hline Localization & 1.07 & 0.14 & 0.83 & 1.39 & 0.583 \\
\hline Neoadjuvant therapy & 1.42 & 0.44 & 0.77 & 2.62 & 0.259 \\
\hline pT category* & 1.45 & 0.23 & 1.06 & 1.99 & 0.020 \\
\hline pN category** & 1.48 & 0.17 & 1.18 & 1.86 & 0.001 \\
\hline pM category & 1.46 & 0.53 & 0.72 & 2.98 & 0.293 \\
\hline $\mathrm{R}$ status** & 2.51 & 0.84 & 1.31 & 4.84 & 0.006 \\
\hline Laurén type & 1.09 & 0.18 & 0.79 & 1.52 & 0.595 \\
\hline rs12268840 $(M G M T)^{*}$ & 1.47 & 0.26 & 1.03 & 2.08 & 0.033 \\
\hline rs9972882 (STARD3)* & 0.66 & 0.13 & 0.44 & 0.98 & 0.041 \\
\hline rs12724079 (ASH1L) & 1.10 & 0.20 & 0.76 & 1.58 & 0.616 \\
\hline rs10423674 (CRTC1) & 1.30 & 0.34 & 0.78 & 2.17 & 0.306 \\
\hline rs1870377 (VEGFR2) & 1.07 & 0.25 & 0.68 & 1.68 & 0.785 \\
\hline rs2898290 (LINC00208) & 0.95 & 0.19 & 0.64 & 1.41 & 0.804 \\
\hline rs2976392 (PSCA) & 1.09 & 0.21 & 0.75 & 1.58 & 0.649 \\
\hline rs2296616 (miR-107) & 1.09 & 0.20 & 0.76 & 1.55 & 0.647 \\
\hline rs10419226 (CRTC1) & 0.79 & 0.18 & 0.50 & 1.23 & 0.294 \\
\hline rs2701108 (TBX5) & 0.80 & 0.17 & 0.52 & 1.22 & 0.306 \\
\hline rs3072 (GDF7) & 1.42 & 0.33 & 0.90 & 2.23 & 0.134 \\
\hline rs4648068 (NFKB1) & 1.33 & 0.28 & 0.88 & 2.01 & 0.173 \\
\hline rs7626449 (DHSs1) & 0.98 & 0.20 & 0.66 & 1.45 & 0.915 \\
\hline pT category** & 1.34 & 0.112 & 1.08 & 1.67 & 0.009 \\
\hline $\mathrm{pN}$ category*** & 1.45 & 0.089 & 1.22 & 1.73 & $<0.001$ \\
\hline $\mathrm{R}$ status*** & 2.69 & 0.264 & 1.61 & 4.51 & $<0.001$ \\
\hline rs12268840 $(M G M T)^{*}$ & 1.33 & 0.142 & 1.01 & 1.75 & 0.045 \\
\hline rs9972882 (STARD3)* & 0.71 & 0.157 & 0.52 & 0.97 & 0.030 \\
\hline
\end{tabular}

\section{DISCUSSION}

In this study we retrieved SNPs from the current literature that were reported to be associated with either BE, GERD, GEJ, or GC. We then analyzed theses SNPs via genotyped and imputed data of curatively resected patients at our medical center. We were able to show that two intron variant SNPs of MGMT and STARD3 were significant predictors for overall survival but not for disease-free survival in gastroesophageal adenocarcinoma. Group TT of rs12268840 showed the highest rate of second primary carcinomas, lowest mRNA expression of $M G M T$ in normal tissue, and worst oncologic outcome, implicating that rs 12268840 could be involved in tumor progression. Group AA of rs 9972882 had the highest rate of distant metastases (pM1), highest mRNA expression of $P G A P 3$, and also worst outcome. The results are consistent with previous works and open a new perspective on clinically relevant germline SNPs that could directly affect carcinogenesis and tumor relapse in upper gastrointestinal cancer.

The SNPs that were selected by this literature-based approach have been identified as relevant for esophageal and gastric adenocarcinoma according to previous genomewide association studies (GWAS) on mostly large collectives. Similar attempts of correlating SNPs with clinical outcome parameters were made by Sung et al. in $2017 .{ }^{8}$ The authors analyzed 11 SNPs that were previously identified as high-evidence genetic susceptibility markers for GC in a former meta-analysis. ${ }^{9}$ Sung et al. were not able to find significant associations of these 11 SNPs with overall survival. However, the authors found subtype-specific associations for GC of the cardia region and diffuse-type GC. 
FIG. 2 a Violin plots for $M G M T$ expression and groups $\mathrm{CC} / \mathrm{CT} / \mathrm{TT}$ in rs12268840 in four different normal tissue types (gastroesophageal junction, esophageal mucosa, esophageal muscularis, and stomach). b Violin plots for $P G A P 3$ expression and groups CC/AC/AA in rs 9972882 in the same four upper gastrointestinal tissues (a)

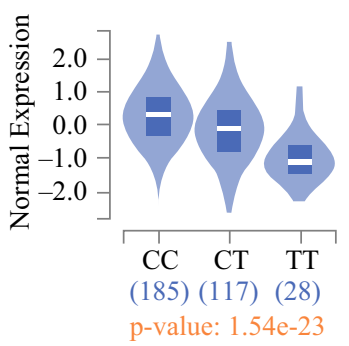

(b)

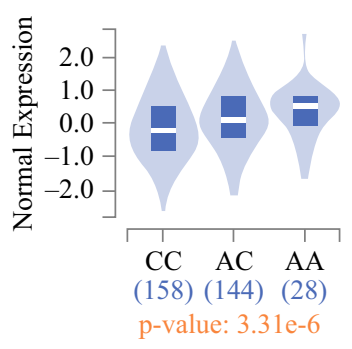

\begin{tabular}{c}
\hline Esophagus \\
Gastroesophageal junction
\end{tabular}

MGMT and rs12268840 (4 different normal tissue types)
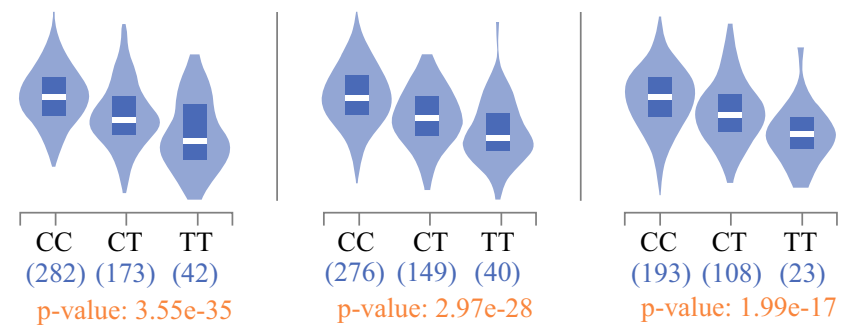

PGAP3 and rs9972882 (4 different normal tissue types)

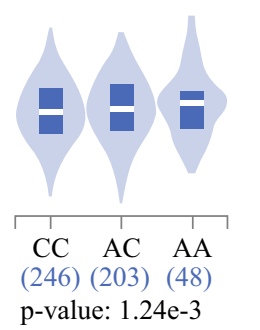

Esophagus
Mucosa

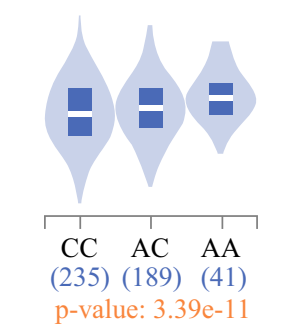

p-value: $3.39 \mathrm{e}-11$

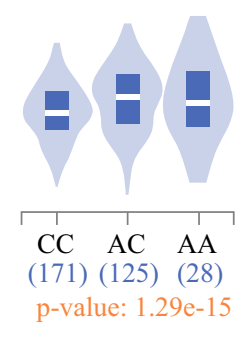

Stomach
In our study, we confirmed usual prognostic factors such as pTNM category and $\mathrm{R}$ status on univariate survival analysis and all variables (except pM category) on multivariate analysis. Based on these results, we assume that this study's validity is ensured concerning the identification of further survival predictors. Only two SNPs, namely rs 12268840 (intron variant of MGMT) and rs9972882 (intron variant of STARD3), were significant on multivariate analysis and will therefore be further discussed. According to the Cox regression model, both SNPs were independent survival predictors next to $\mathrm{pT} / \mathrm{pN}$ categories and $\mathrm{R}$ status.

SNP rs12268840 is located on chromosome 10 and is known as an intron variant of $M G M T$ that encodes for the $O$-6-methylguanine-DNA methyltransferase (abbreviated as MGMT). DNA methylation is one of the most important types of epigenetic modification and plays a crucial role in carcinogenesis. ${ }^{10}$ MGMT is a DNA-repair enzyme that protects cells from carcinogenic effects by removing DNA adducts of alkylating agents from the $O-6$ position of guanine. ${ }^{11}$ Thus, studies have shown that loss of MGMT leads to an increased carcinogenic risk in mice. ${ }^{12}$ For gastric cancer, Zhang et al. were able to demonstrate that $M G M T$ silencing via promoter hypermethylation was significantly associated with an increased risk of GC. ${ }^{13}$ Other works have shown that reduced expression of MGMT in esophageal adenocarcinoma organoids led to sensitivity towards temozolomide and taxane agents. ${ }^{14}$ The study by Doecke et al. that initiated the inclusion of rs12268840 to this literature-based approach found that homozygous carriers of rs12268840 (i.e., group TT) with frequent acid reflux had a significantly higher risk of GEJ. ${ }^{15}$

Our cis-eQTL analysis has shown that, in upper gastrointestinal tissues of healthy probands, group TT expressed the least MGMT mRNA in comparison with groups CC and CT (Fig. 2a). Furthermore, within the groups of rs 12268840 , there were significant different rates of second primary carcinomas (SPC). SPCs are defined as any type of malignant disease that was reported in the patient's past medical history and was treated beforehand. ${ }^{16}$ Patients in group TT had the highest rate of SPC, while patients in group TC and CC had lower rates of SPC and significantly higher median OS. We interpret these results as group TT possibly having had a lack of MGMT to fight malignant diseases in the past medical history as well as in the postoperative follow-up. This may have led to a worse oncologic outcome in general.

Although SNP rs9972882 most likely qualifies as an intron variant of STARD3, it proved to be a significant ciseQTL for PGAP3 according to mRNA expression in normal upper gastrointestinal tissue. In contrast, rs 9972882 was not a significant eQTL for neighboring genes STARD3 or ERBB2 (Her2/neu).

$P G A P 3$ transcribes the post-GPI attachment to proteins phospholipase 3 , which is an enzyme for fatty acid remodeling of glycosylphosphatidylinositol (GPI)-anchored proteins (abbreviated as GPI-APs). GPI-APs are 
glycoproteins that are anchored to the outer layer of the plasma membrane and therefore exposed on the cell surface. Precisely, PGAP3 is responsible for the removal of unsaturated fatty acids at the sn-2 position of GPI-APs. Germline mutations of PGAP3 lead to a subtype of hyperphosphatemia and intellectual disabilities, also known as Mabry syndrome type $4 .{ }^{17}$ Kwon et al. ${ }^{18}$ have found that PGAP3 is co-amplified (together with STARD3, GRB7, and MIEN1) with ERBB2(Her2/neu) in esophageal and gastric cancer. Murakami et al. ${ }^{19}$ were able to show that PGAP3 knockout mice had enhanced responses to alloreactive and antigen-specific stimuli. It is clear that GPI-APs play important roles in many biological processes such as signal transduction and cell-cell interaction. Our data could be interpreted that overexpression of PGAP3 within group AA of rs 9972882 represents a malfunctioning of GPI anchoring.

The classification of gastric cancer by The Cancer Genome Atlas (TCGA) Research Network into EpsteinBarr virus (EBV)-positive, microsatellite instability (MSI)high, genomically stable (GS), and chromosomally instable (CIN) subtypes has drawn attention to a general molecular differentiation of gastric cancer. ${ }^{20}$ The differentiation of molecular subtypes could be especially relevant for the prediction of potential susceptibility for (neo-)adjuvant treatment. ${ }^{21}$ So far, the CIN subtype was encountered more frequently in the gastroesophageal junction and cardia, ${ }^{22}$ which was also the most frequent anatomic location of gastric adenocarcinoma in this study group. Due to its role as a DNA-repair enzyme, MGMT and its dysfunction may represent another aspect of the CIN subtype demonstrated by TCGA. However, tumor tissue and sequencing patterns would be necessary to properly investigate the effect of MGMT expression (and PGAP3, respectively) on actual prognosis for the separate TCGA subtypes. For this reason, genomic and tumorspecific data analysis will be necessary to infer that these results are generally applicable to all gastroesophageal cancer subtypes.

This study is based on a data collection of numerous clinical variables to enable a profound analysis of essential prognostic factors accompanied by reliable survival data from a systematic follow-up. Moreover, our analyses allow a linkage and translational approach to investigate histopathological, oncological, and genotyped features of upper gastrointestinal tumors. The ultimate goal of this study was to identify those genetic alterations that actually have a clinical implication in terms of a significantly different prognosis. However, it is necessary to remark that there may be a potential selection bias concerning those SNPs that could be of interest but were not investigated due to missing reports in the literature. Another limitation of this study is that both GEJ and GC were analyzed in the same collective in terms of testing for specific SNP markers. However, we were able to prove beforehand that both groups did not differ in decisive outcome parameters. Last but not least, the separate analysis of both groups GEJ and GC did not show any significant differences, neither for overall nor for disease-free survival. Previous works by our own group have demonstrated the comparability of both cancer entities regarding long-term oncological outcome. ${ }^{23}$ This circumstance may also be in accordance with the insights by TCGA that distant esophageal adenocarcinoma and chromosomally unstable gastric adenocarcinoma (the most frequent TCGA subtype of gastric cancer) share many genomic amplifications and could possibly be considered a single disease entity. ${ }^{24}$

By correlating the above-mentioned SNPs with our clinical data, we identified two clinically relevant SNPs. Our data suggest that the DNA-repair enzyme MGMT is relevant in gastroesophageal cancer and that PGAP3 is a potentially novel agent in carcinogenesis. In the future, it will be necessary to reproduce these results for larger and more homogeneous samples. Eventually, in vitro studies are required to comprehend the molecular mechanisms of $M G M T$ and $P G A P 3$.

FUNDING Open Access funding enabled and organized by Projekt DEAL.

DISCLOSURE None of the authors has any financial conflicts of interest to declare.

Supplementary Information The online version contains supplementary material available at https://doi.org/10.1245/s10434021-10771-y.

OPEN ACCESS This article is licensed under a Creative Commons Attribution 4.0 International License, which permits use, sharing, adaptation, distribution and reproduction in any medium or format, as long as you give appropriate credit to the original author(s) and the source, provide a link to the Creative Commons licence, and indicate if changes were made. The images or other third party material in this article are included in the article's Creative Commons licence, unless indicated otherwise in a credit line to the material. If material is not included in the article's Creative Commons licence and your intended use is not permitted by statutory regulation or exceeds the permitted use, you will need to obtain permission directly from the copyright holder. To view a copy of this licence, visit http://creativecommons. org/licenses/by/4.0/.

\section{REFERENCES}

1. Bray F, Ferlay J, Soerjomataram I, Siegel RL, Torre LA, Jemal A. Global cancer statistics 2018: GLOBOCAN estimates of incidence and mortality worldwide for 36 cancers in 185 countries. CA Cancer J Clin. 2018;68(6):394-424. https://doi.org/10. 3322/caac.21492. 
2. Marano L, Polom K, Patriti A, et al. Surgical management of advanced gastric cancer: an evolving issue. Eur J Surg Oncol. 2016;42(1):18-27. https://doi.org/10.1016/j.ejso.2015.10.016.

3. Coleman HG, Xie SH, Lagergren J. The epidemiology of esophageal adenocarcinoma. Gastroenterology. 2018;154(2):390-405. https://doi.org/10.1053/j.gastro.2017.07. 046.

4. Argyrou A, Legaki E, Koutserimpas C, et al. Risk factors for gastroesophageal reflux disease and analysis of genetic contributors. World J Clin Cases. 2018;6(8):176-82. https://doi.org/10. 12998/wjcc.v6.i8.176.

5. Abdi E, Latifi-Navid S, Zahri S, Yazdanbod A, Pourfarzi F. Risk factors predisposing to cardia gastric adenocarcinoma: insights and new perspectives. Cancer Med. 2019;8(13):6114-26. http s://doi.org/10.1002/cam4.2497.

6. Auton A, Abecasis GR, Altshuler DM, et al. A global reference for human genetic variation. Nature. 2015;526(7571):68-74. h ttps://doi.org/10.1038/nature15393.

7. Brierley J, Gospodarowicz M, Wittekind C. TNM classification of malignant tumours 2017. 8th Ed.; 2017.

8. Sung $\mathrm{H}, \mathrm{Hu} \mathrm{N}$, Yang $\mathrm{HH}$, et al. Association of high-evidence gastric cancer susceptibility loci and somatic gene expression levels with survival. Carcinogenesis. 2017;38(11):1119-28. http s://doi.org/10.1093/carcin/bgx090.

9. Mocellin S, Verdi D, Pooley KA, Nitti D. Genetic variation and gastric cancer risk: a field synopsis and meta-analysis. Gut. 2015;64(8):1209-19. https://doi.org/10.1136/gutjnl-2015309168.

10. Kulis M, Esteller M. DNA methylation and cancer. Vol 70. Adv Genet; 2010. doi:https://doi.org/10.1016/B978-0-12-380866-0.60 $002-2$

11. Qu Y, Dang S, Hou P. Gene methylation in gastric cancer. Clin Chim Acta. 2013;424:53-65. https://doi.org/10.1016/j.cca.2013. 05.002 .

12. Shiraishi A, Sakumi K, Sekiguchi M. Increased susceptibility to chemotherapeutic alkylating agents of mice deficient in DNA repair methyltransferase. Carcinogenesis. 2000;21(10):1879-83. https://doi.org/10.1093/carcin/21.10.1879.

13. Zhang Z, Xin S, Gao M, Cai Y. Promoter hypermethylation of MGMT gene may contribute to the pathogenesis of gastric cancer. Med (USA). 2017. https://doi.org/10.1097/MD. 0000000000006708.

14. Jammula SG, Biasci D, Devonshire G, et al. Identification of subtypes of Barrett's esophagus and esophageal adenocarcinoma based on DNA methylation profiles and integration of transcriptome and genome data. Gastroenterology. 2020;158(6):16821697.e1. https://doi.org/10.1053/j.gastro.2020.01.044.

15. Doecke J, Zhao ZZ, Pandeya N, et al. Polymorphisms in MGMT and DNA repair genes and the risk of esophageal adenocarcinoma. Int J Cancer. 2008;123(1):174-80. https://doi.org/10.1002/ ijc. 23410 .

16. Jung JO, Schulz ER, Nienhüser $\mathrm{H}$, et al. Characteristics and prognostic factors of metachronous second primary upper gastrointestinal cancer. J Surg Res. 2021;258:254-64. https://doi.org/ 10.1016/j.jss.2020.08.075.

17. Howard MF, Murakami Y, Pagnamenta AT, et al. Mutations in PGAP3 impair GPI-anchor maturation, causing a subtype of hyperphosphatasia with mental retardation. Am J Hum Genet. 2014;94(2):278-87. https://doi.org/10.1016/j.ajhg.2013.12.012.
18. Kwon MJ, Kim RN, Song K, et al. Genes co-amplified with ERBB2 or MET as novel potential cancer-promoting genes in gastric cancer. Oncotarget. 2017;8(54):92209-26. https://doi.org/ 10.18632/oncotarget.21150.

19. Murakami H, Wang $Y$, Hasuwa $H$, Maeda $Y$, Kinoshita $T$, Murakami Y. Enhanced response of T lymphocytes from Pgap3 knockout mouse: insight into roles of fatty acid remodeling of GPI anchored proteins. Biochem Biophys Res Commun. 2012;417(4):1235-41. https://doi.org/10.1016/j.bbrc.2011.12. 116.

20. Bass AJ, Thorsson V, Shmulevich I, et al. Comprehensive molecular characterization of gastric adenocarcinoma. Nature. 2014;513(7517):202-9. https://doi.org/10.1038/nature13480.

21. Kohlruss M, Grosser B, Krenauer M, et al. Prognostic implication of molecular subtypes and response to neoadjuvant chemotherapy in 760 gastric carcinomas: role of Epstein-Barr virus infection and high- and low-microsatellite instability. J Pathol Clin Res. 2019;5(4):227-39. https://doi.org/10.1002/cjp2.137.

22. Wang Q, Liu G, Hu C. Molecular classification of gastric adenocarcinoma. Gastroenterol Res. 2019;12(6):275-82. https://doi. org/10.14740/gr1187.

23. Sisic L, Strowitzki MJ, Blank S, et al. Postoperative follow-up programs improve survival in curatively resected gastric and junctional cancer patients: a propensity score matched analysis. Gastric Cancer. 2018;21(3):552-68. https://doi.org/10.1007/s10 120-017-0751-4.

24. Kim J, Bowlby R, Mungall AJ, et al. Integrated genomic characterization of oesophageal carcinoma. Nature. 2017;541(7636):169-74. https://doi.org/10.1038/nature20805.

25. Sung $\mathrm{H}$, Yang $\mathrm{HH}$, Zhang $\mathrm{H}$, et al. Common genetic variants in epigenetic machinery genes and risk of upper gastrointestinal cancers. Int J Epidemiol. 2015;44(4):1341-52. https://doi.org/10. 1093/ije/dyv050.

26. Berardi R, Torniai $\mathrm{M}$, Partelli $\mathrm{S}$, et al. Impact of vascular endothelial growth factor (VEGF) and vascular endothelial growth factor receptor (VEGFR) single nucleotide polymorphisms on outcome in gastroenteropancreatic neuroendocrine neoplasms. Nie D, ed. PLoS One. 2018;13(5):e0197035. doi:h ttps://doi.org/10.1371/journal.pone.0197035

27. Gharahkhani P, Fitzgerald RC, Vaughan TL, et al. Genome-wide association studies in oesophageal adenocarcinoma and Barrett's oesophagus: a large-scale meta-analysis. Lancet Oncol. 2016;17(10):1363-73. https://doi.org/10.1016/S1470-2045(16)30 240-6.

28. Qiu Y, Zhang ZY, Du WD, et al. Association analysis of ERBB2 amplicon genetic polymorphisms and STARD3 expression with risk of gastric cancer in the chinese population. Gene. 2014;535(2):225-32. https://doi.org/10.1016/j.gene.2013.11.030.

29. Levine DM, Ek WE, Zhang R, et al. A genome-wide association study identifies new susceptibility loci for esophageal adenocarcinoma and Barrett's esophagus. Nat Genet. 2013;45(12):1487-93. https://doi.org/10.1038/ng.2796.

Publisher's Note Springer Nature remains neutral with regard to jurisdictional claims in published maps and institutional affiliations. 